\title{
Numerically simulated 3D-structures of the fluctuating fields
}

\author{
A.E.Filippov \\ Donets'k Institute for Physics and Engineering, \\ 83114 Donets'k, Ukraine
}

Received February 8, 2001

\begin{abstract}
Numerical simulation of a structure of the fluctuating field appearing at a kinetic stage of the phase transition is performed for the systems with scalar and two-component (complex) order parameters. It is found that the ordering process is accompanied by a large-scale filamentary structure of the fluctuating field. The dense local groups and chains of the nuclei are created in the system. A complicated topology of the ordering is found in the system with complex order parameter. This system creates specific "toroidal" vortices. Kinetic scenarios in 3D-space are compared with the 2D- and 1D projections of the analogous structures previously found.
\end{abstract}

Key words: phase transitions, fluctuations, nucleation, kinetics

PACS: $64.60 . A k, 64.60 . Q b$

\section{Introduction}

For the last few years the kinetics of the ordering at phase transitions and phase separation has attracted strong attention. Modern computers open up principally new possibilities in the field. To perform an analytical study, it was necessary to apply a lot of approximations (for example, the idea of a spherical critical nucleus with a specific radius determined by a balance of energy between a gain of energy in the ordered state and an energy of a "surface tension" [1-3]).

The current level of computing makes it possible, in principle, to accomplish a direct Brownian dynamic (BD) simulation of complex systems. A number of the examples of the BD simulation can be found in the literature (see, for example [415]). This approach is not limited to the low space dimensionalities $d \leqslant 3$ and can be easily generalized onto $d=3$. More important here is a limited number of the interacting particles $N$.

Really, the number of elementary interactions grows as a square of the value $N$. As a rule, nontrivial results can be obtained by the BD approaches at the number $N$ close to $10^{3}$. It corresponds to the $N^{2}=10^{6}$ elementary interactions. It stimulates the search for the analogous structures by an alternative kinetic approach, based on 
a study of the continuous fields. The main approximation in this approach consists of a transformation of the problem to a continuous one, based on the collective-field description [16-20].

However, to describe the problem in 3D-space in the kinetic approach one needs a number of elementary cells in the grid close to $N_{x}=N_{y}=N_{z}=10^{2}$. In other words, a total number of the sells $N_{x}^{3}=10^{6}$ in the system has the same order as in the BD. So, the numerical difficulties are comparable in the both approaches.

A few years ago, we stated some suppositions regarding a structure of the fluctuating order parameter field in the physical 3D-space [32-39]. But,these suppositions have not been tested yet by a direct numerical simulation. However, among other reasons, such a test is interesting for a development of the kinetic approach by itself. In this note some results of such a study are presented for the following models often used in the theory of phase transition:

model 1 - the model with scalar order parameter;

model 2 - continuously degenerated model with two-component (complex) order parameter (which is a prototype of the models describing vector systems and the systems with the interacting order parameters, as well as the superconductors).

\section{Kinetic equations and the free energy functionals}

The order parameter evolution in the non-equilibrium systems can be described by the generalized Landau-Khalatnikov equation [21-23]

$$
\partial \boldsymbol{\varphi}(\mathbf{r}, t) / \partial t=-\gamma(\delta \mathbf{H}[\boldsymbol{\varphi}(\mathbf{r}, t)] / \delta \boldsymbol{\varphi}) .
$$

Here $\mathbf{H}$ is the Ginzburg-Landau functional; $\gamma$ is a positive kinetic coefficient, $\boldsymbol{\varphi}(\mathbf{r}, t)$ is a vector order parameter field. Let us limit ourselves to the following form of the Ginzburg-Landau functional:

$$
\mathbf{H}[|\boldsymbol{\varphi}(\mathbf{r})|]=\int \mathrm{d}^{\mathrm{d}} \mathbf{r}\left[|\nabla \boldsymbol{\varphi}(\mathbf{r})|^{2} / 2+f(|\boldsymbol{\varphi}(\mathbf{r})|)\right]=\int \mathrm{d}^{\mathrm{d}} \mathbf{r} F(|\boldsymbol{\varphi}(\mathbf{r})|) .
$$

There are many different problems that can be reduced to this form of the functional. In particular, it is used in the theory of binary solvents, uniaxial magnets, martensits, superconductors, etc. [16-18,24-28].

In model 1 for the first order phase transition [1,2,16-18] the local density of free energy can be reduced to the two standard expansions over the unique (scalar) combination $\varphi(\mathbf{r})$ of the order parameter components:

$$
\begin{aligned}
& f(\varphi(\mathbf{r}))=\tau \varphi(\mathbf{r})^{2} / 2-2 \alpha \varphi(\mathbf{r})^{3} / 3+\beta \varphi(\mathbf{r})^{4} / 4 \\
& f(\varphi(\mathbf{r}))=\tau \varphi(\mathbf{r})^{2} / 2-\alpha \varphi(\mathbf{r})^{4} / 4+\beta \varphi(\mathbf{r})^{6} / 6 .
\end{aligned}
$$

At positive constants $\tau, \alpha$ and $\beta$, as well as $\tau \beta<\alpha^{2}$ the functions $f(\varphi(\mathbf{r}))$ have the metastable minimum at $\varphi(\mathbf{r})=\varphi_{0}=0$ and global ones in $\varphi_{ \pm}^{2}$ respectively:

$$
\varphi_{+}=\left[\alpha+\left(\alpha^{2}-\tau \beta\right)^{1 / 2}\right] / 2 ; \quad \varphi_{ \pm}^{2}=\left[\alpha-\left(\alpha^{2}-\tau \beta\right)^{1 / 2}\right] / \beta .
$$


In the case 2 we use the standard model $\varphi^{4}(\mathbf{r})$ for the two-component (complex) order parameter

$$
f(|\boldsymbol{\varphi}(\mathbf{r})|)=\tau|\boldsymbol{\varphi}(\mathbf{r})|^{2}+\beta\left(|\boldsymbol{\varphi}(\mathbf{r})|^{2}\right)^{2} / 4 .
$$

The new phase nucleus forms from the fluctuations that (in kinetic approach) are created by the random (Gaussian) source $\zeta(\mathbf{r}, t)$ that has to be included into the equation (1):

$$
\left\langle\zeta_{j}((\mathbf{r}, t)\rangle=0 ; \quad\left\langle\zeta_{j}(\mathbf{r}, t) \zeta_{j^{\prime}}\left(\mathbf{r}^{\prime}, t\right)\right\rangle=D \delta_{j j^{\prime}} \delta\left(\mathbf{r}-\mathbf{r}^{\prime}\right) \delta\left(t-t^{\prime}\right) .\right.
$$

In accordance with the general hypothesis of the fluctuation theory [16-18] it is supposed here that different components of the order parameter fluctuate independently. It is reflected by the factor $\delta_{j j^{\prime}}$ in the correlator $\left\langle\zeta_{j}(\mathbf{r}, t) \zeta_{j^{\prime}}\left(\mathbf{r}^{\prime}, t\right)\right\rangle$ in a case of the two components of the order parameter $j=1,2$.

\section{General notes and the expected results}

Different versions of the modified equation (1) were intensively used to simulate the localized nonlinear excitations and nucleation [29-38] in 1 and 2 dimensions. In particular, the analytical and numerical evidences were found that the critical nuclei have to appear (even in isotropic 3D space!) from low-dimensional folds of the fluctuation field density.

Moreover, the critical configuration of the order parameter does not have any specific form and its density in the maximum $\varphi(\mathbf{r})$ can be essentially lower than a value $\varphi_{0}(\mathbf{r})$ of the equilibrium order parameter. In fact, such a density fold is a particular realization of the large-scale (mesoscopic) fluctuation. A distribution in the space of the growing (super-critical) nuclei "memorizes" the preliminary density folds. So, it is expected that a fluctuating order parameter forms some large-scale "filamentary" structure. Such a structure has been observed numerically in 2D space [32]. It has been shown that this structure is a dissipative attractor corresponding to a minimal dissipation rate [33]. In the present study we confirm all these results for the realistic $3 \mathrm{D}$ system.

The most interesting feature of the model 2 see formula (5) is the appearance of the vortex order parameter configurations. In the 2D systems these configurations normally appear due to independent fluctuations of the two order components $[39,40]$. In fact, the vortices are created in the points of mutual intersection of the lines of zero of the order parameter components. In the 3D-space such an intersection is possible for the planes only. So, a creation of the usual (one-dimensional) vortex line in the 3D-system is not trivial and needs a special study.

A problem like this was discussed in relation to the vortex formation in the systems with vector ("nontrivial") pairing [41], but in another context. The number of the components of the order parameter in these systems is not less than 4 . Even for the planar system the spontaneous cross-section of 4 zero-lines in the sole point is incredible. It has been shown, however, that there is a stable kinetic process leading to a creation of the one-dimensional channels with the lowered order parameter. 
These channels play the role of the so-called "precursors" for the vortices. They are no zero-dimensional point-like singularities, but at some conditions these channels regularly assemble into the singular vortices.

Something similar is presented in this paper for the 3D systems with a complex order parameter. The growing total order parameter captures here the cavities appearing near the surfaces of the zeros of its components. One can prove that there is a "current" of the order parameter that is determined by the standard combination of its components and their gradients:

$$
\mathbf{J}=\left(\varphi_{1} \nabla \varphi_{2}-\varphi_{2} \nabla \varphi_{1}\right)
$$

Inside the cavity an effective field $\mathbf{H}$ exists that one can associate with the current $\mathbf{J}$. This field is created by the $\operatorname{rot} \mathbf{J}$ :

$$
\mathbf{H}=\operatorname{rot} \mathbf{J}=\operatorname{rot}\left(\varphi_{1} \nabla \varphi_{2}-\varphi_{2} \nabla \varphi_{1}\right)
$$

This current creates the toroidal configurations of the effective "field" $\mathbf{H}$. There are at least 2 spots of the flux $\mathbf{H}$ with mutually opposite directions of the vector $\mathbf{H}$ in a cross-section of such a torus by a plane. It has to be noted that for the superconducting systems (containing the gauge filed interacting with the order parameter) the vector $\mathbf{H}=\operatorname{rot} \mathbf{J}$ describes a creation of the physical magnetic field inside the system in the course of the phase transition [40]. In the 2D space the well known vortex-antivortex configurations correspond to the structure described.

It is also well known that appearing in the kinetics these pairs collapse and annihilate in the course of relaxation. Physically it corresponds to a lowering of the surface energy near such a pair. This energy is created by the non-zero impacts coming from the gradient terms $\delta E=\int \mathrm{d}^{d} \mathbf{r}|\nabla \boldsymbol{\varphi}(\mathbf{r})|^{2} / 2$. An analogous process takes place in the 3D space. One can expect that the annihilation of the pairs corresponds here to the gathering and thus to the disappearance of the toroidal fluxes. This process lowers the surface boundary by a smoothing of the domain boundaries as well.

\section{Results and discussion}

All the structures, described qualitatively above, have been obtained by a direct simulation of the ordering kinetics (in numerical experiments that generalize our previous studies into 3D space).

The results are summarized in figures $1-7$. The results have been obtained for the systems containing more than $10^{6}$ cells of the numerical grid (as a rule, $N=$ $128 \times 128 \times 128)$. To control the procedure, the number of the cells was varied in a wide interval $(256 \times 256 \times 32$, or $256 \times 128 \times 64$, etc $)$. The calculations are performed with the periodic boundary conditions. It reduces the influence of the boundaries to a minimum.

A typical developed "filamentary structure" is shown in figure 1. It is impossible to present the density $\varphi(\mathbf{r})$ in the 3D-space; a surface of a constant value $\varphi(\mathbf{r})=$ 


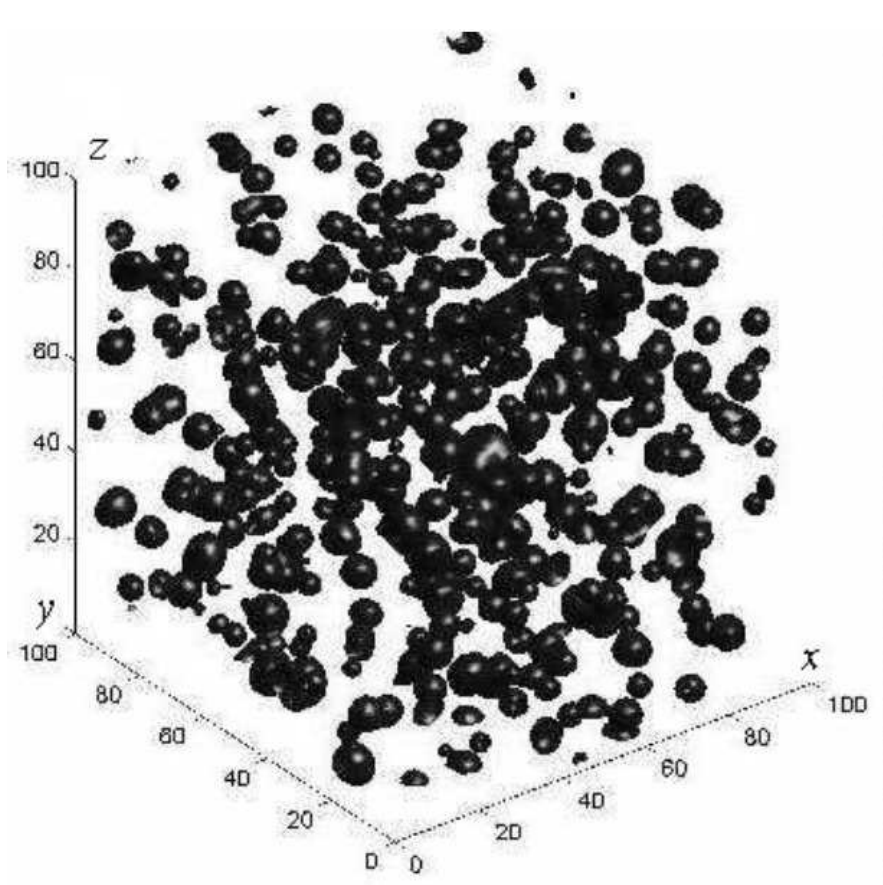

Figure 1. Typical developed "filamentary" structure of the order parameter field in scalar model. The distribution of the $\varphi(\mathbf{r})$ in a space is shown by means of the surface of a constant value of the $\varphi(\mathbf{r})\left(\varphi(\mathbf{r})=0.9 \varphi_{0}\right.$, where $\varphi_{0}=1$ is the equilibrium order parameter value).

const is shown here. The particular value for the constant is taken to be equal to $\varphi=0.9 \varphi_{\max }$ of a maximal value $\varphi_{\max }$. In this particular case the maximum $\varphi_{\max }$ of the distribution coincides $\varphi(\mathbf{r})$ approximately with the equilibrium value $\varphi_{\max } \approx \varphi_{0}$. The results in figure 1 look qualitatively close for the both of the models 3 and 4 and are valid for the space distribution of the modulus of the order parameter in model (4). The distributions have been obtained at the same values of the parameters as in the papers $[32,33]$.

The structure of the order parameter $\varphi(\mathbf{r})$ can be described in the terms of two-point correlation function

$$
\mathrm{G}(\mathbf{r})=\langle\varphi(\mathbf{r}) \varphi(0)\rangle=\int \mathrm{d}^{\mathrm{d}} \mathbf{r}^{\prime} \varphi\left(\mathbf{r}^{\prime}\right) \varphi\left(\mathbf{r}^{\prime}-\mathbf{r}^{\prime}\right) / V .
$$

Numerically this correlator can be calculated at an arbitrary time moment for an instant data array $\varphi(\mathbf{r})$. The surface $G(\mathbf{r})=$ const in this case is not convenient to present 3D function $G(\mathbf{r})$. It shadows an internal structure of the $G(\mathbf{r})$. It is more informative here to plot its cross-section by one or two planes (like $z=$ const and sometimes also $y=$ const).

A sequence of the (consecutive in time) cross-sections of the $G(\mathbf{r})$ by the plane $z=$ const is shown in figure 2. The time steps for the plots are chosen to be equal $t=1 / \gamma, 10 / \gamma, 20 / \gamma, 30 / \gamma$ respectively. The density $G(\mathbf{r})$ is shown by the grayscale map. The map is normalized on the maximum value of $G$ at $\mathbf{r}=0: G(\mathbf{r}) \rightarrow$ $G(\mathbf{r}) / G(0)$. Black colour corresponds to the $G(0)$. 


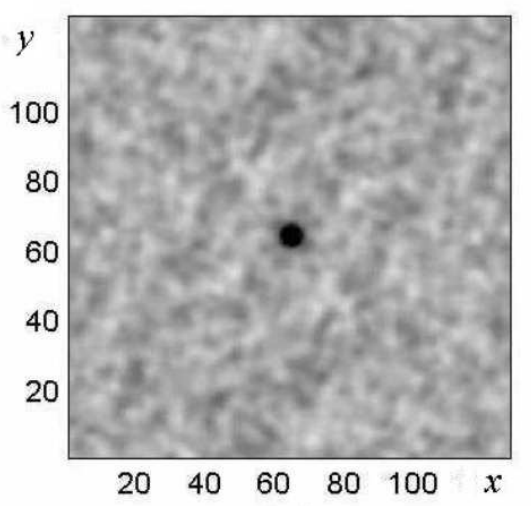

(a)

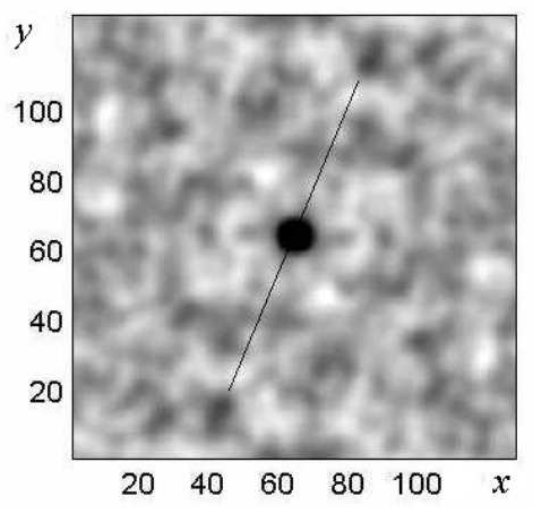

(c)

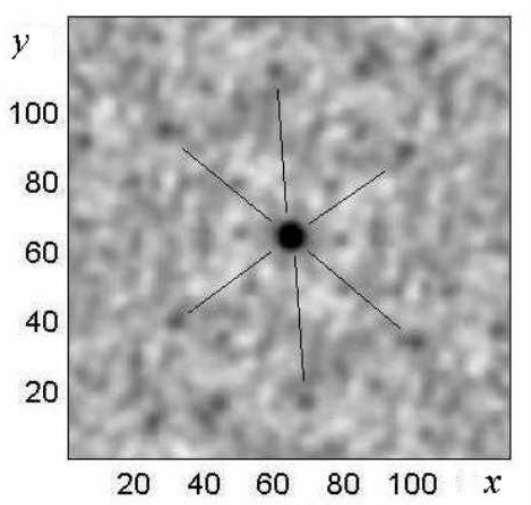

(b)

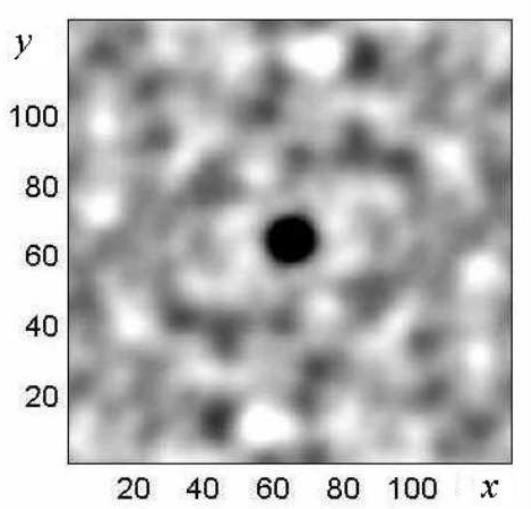

(d)

Figure 2. The consequent evolution stages of the space distribution of two-point correlation function $G(\mathbf{r})$. The $2 \mathrm{D}$-distribution $G(\mathbf{r})$ is calculated after a crosssection of the 3D-density $\varphi(\mathbf{r})$ by an arbitrary chosen plane with $z=$ const the value $G(\mathbf{r})$ is normalized on its value in maximum and presented in a form of the gray-scale map. The maximum $\mathrm{G}(0)=1$ corresponds to the black colour.

The initial distribution of the $G(\mathbf{r})$ with the only narrow black maximum in the center is close to the one for the Gaussian noise. An expansion of this maximum corresponds to a local growth of the nuclei and to an increase of the correlation radius in the system. One can also note some secondary maximums. Let us denote them as $G_{\max }$. In figure 3a, the relative value of the secondary maximums $R=G_{\max } / G(0)$ is shown in the cross-section of the $G(\mathbf{r})$ by a couple of the planes $z=$ const and $y=$ const. The secondary maximums are separated from the central one. It corresponds to a creation of the structure with a specific scale.

The relation between the maximums:

$$
R=\mathrm{G}_{\max } / \mathrm{G}(0)
$$

can be interpreted as a quantitative parameter. In fact, it is some "order parameter" describing the secondary structure appearing at a kinetic stage of the ordering. Its time dependence is shown in figure $3 \mathrm{~b}$. 


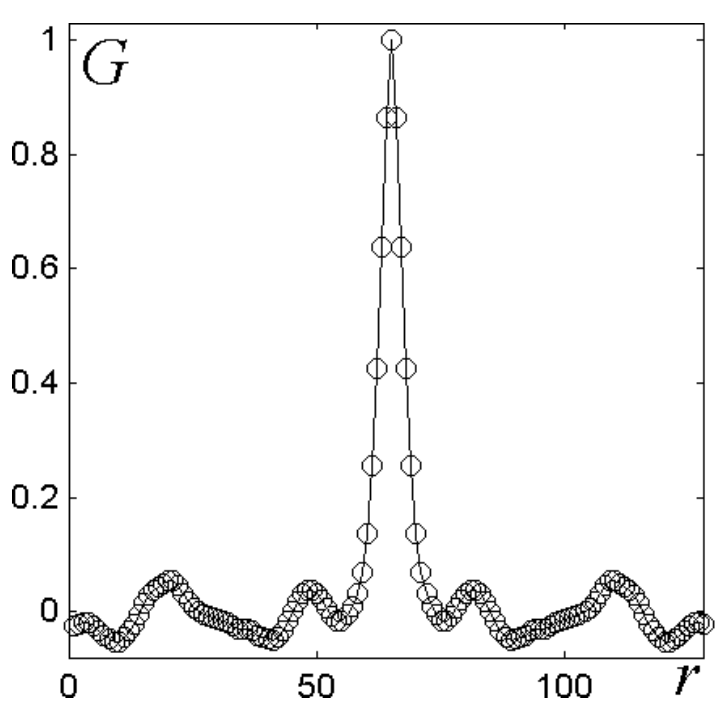

(a)

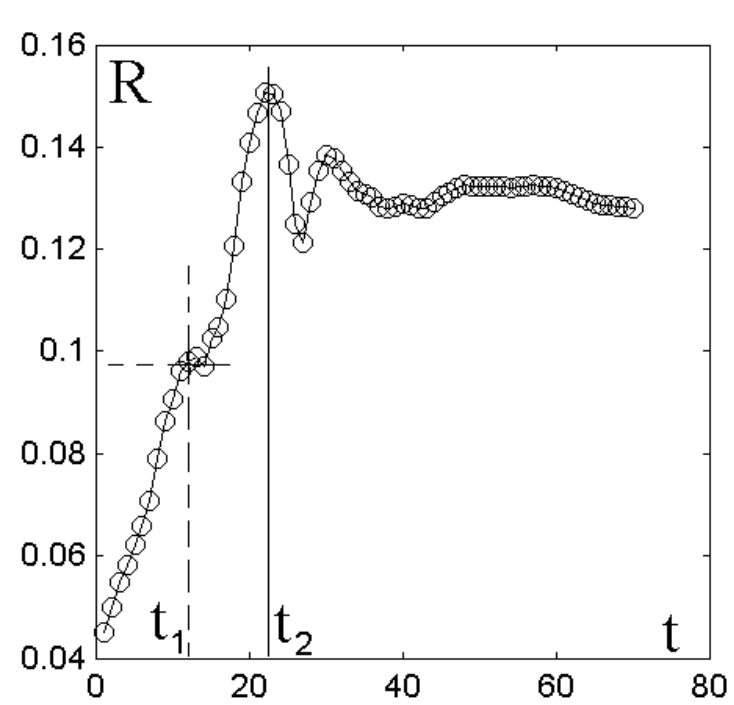

(b)

Figure 3. Time evolution of the relative value of the secondary maxima of the correlation function $G(\mathbf{r})$ : (a) - a cross-section of the correlation function $G(\mathbf{r})$ by the planes $z=$ const, $y=$ const at a moment of maximal development of the "filamentary" structure; (b) - the evolution of a normalized value of the secondary maxima of the correlation function $G(\mathbf{r})$ with the time.

Two peculiarities on the curve $R(t)$ are seen in this plot. These peculiarities correspond to the characteristic stages of the evolution of the secondary structure. The first of them (near $t_{1}$ ) corresponds to the correlation function shown in figure $2 \mathrm{~b}$. This time, the critical nuclei are formed (locally) everywhere in the system. They interact with the aid of the gradient terms. One can observe here a tendency to the 6 -fold symmetry in the secondary maxima of the $G(\mathbf{r})$. It is a typical one for the compact packing of the spheres. The directions from a center to these maximums are marked in the plot of figure $2 \mathrm{~b}$ by the straight lines.

Later (an interval between $t_{1}$ and $t_{2}$ in figure $2 \mathrm{~b}$ ) a quick growth of the supercritical nuclei is observed in the system accompanied by the appearance of an essentially anisotropic structure. At this time, the couple of the maximums of the correlation function $G(\mathbf{r})$ start being selected between the other ones. Such a pair of the maximums is connected by a straight line in figure 2c. The system holds firmly this direction and does not change it up to a completely ordered state (see figure $2 \mathrm{~d}$ ). The filaments of the "glued" nuclei become comparable with a size of the data array.

The rate of the energy dissipation

$$
W=\langle\partial \mathbf{H}[\varphi(\mathbf{r}, t)] / \partial t\rangle
$$

is an important characteristic of the process. Comparing this definition with the kinetic equation, one can obtain a following relation

$$
\begin{aligned}
W & =\langle\partial \varphi(\mathbf{r}, t) / \partial t(\delta \mathbf{H}[\varphi(\mathbf{r}, t)] / \delta \varphi)\rangle \\
& =-\gamma \int \mathrm{d}^{\mathrm{d}} \mathbf{r}^{\prime}(\delta \mathbf{H}[\varphi(\mathbf{r}, t)] / \delta \varphi)^{2} / V,
\end{aligned}
$$




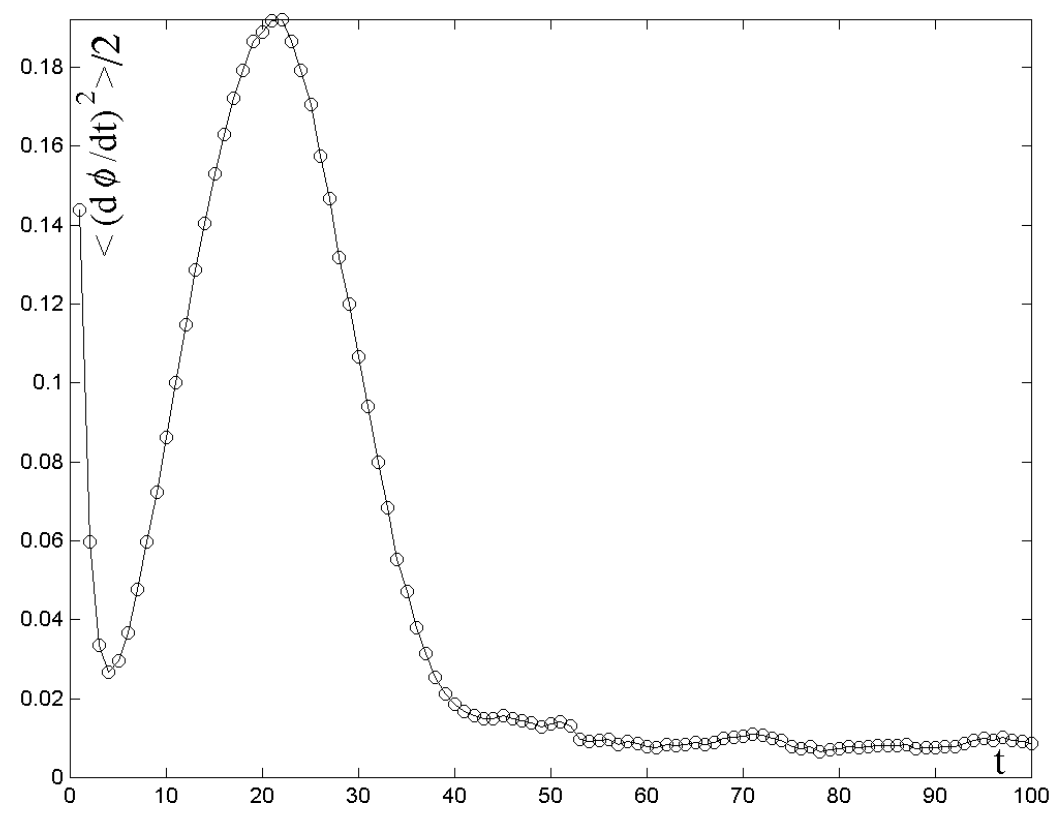

Figure 4. The variation of the dissipation rate $W(t)$ during an ordering process, calculated for the scalar field.

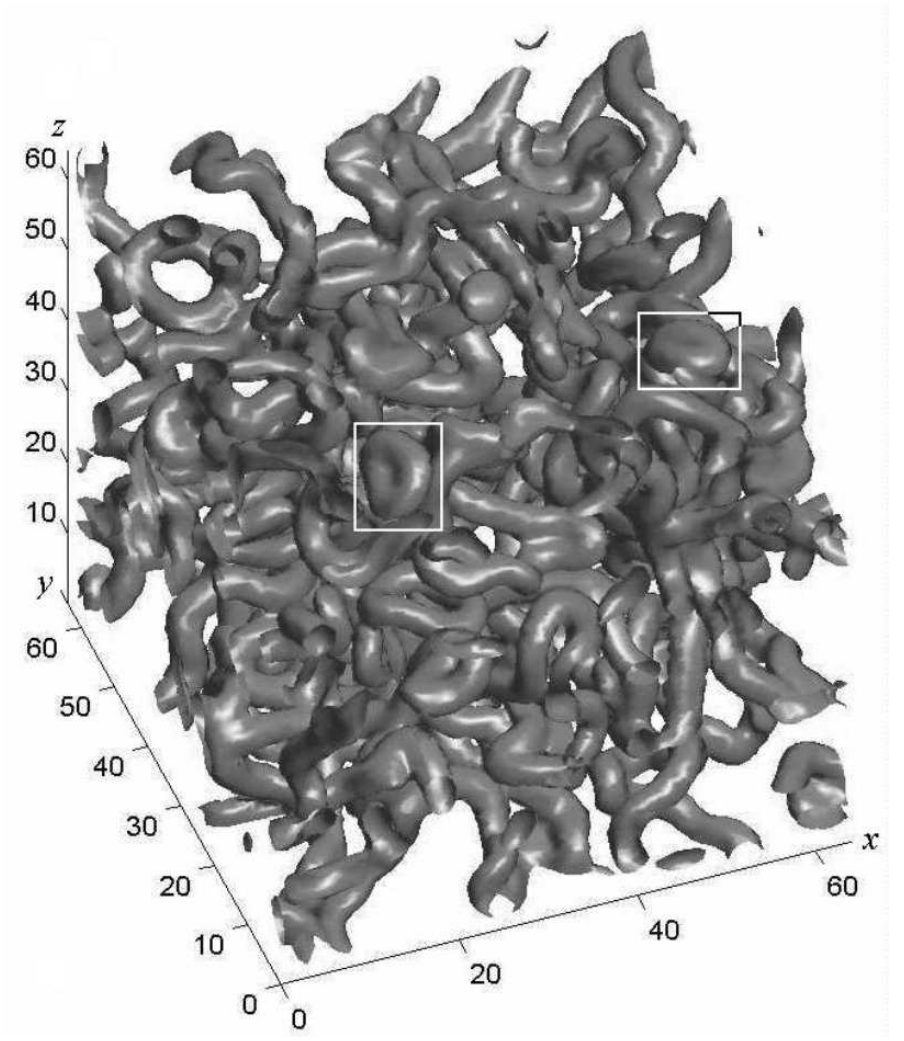

Figure 5. A fragment of a large-scale structure of the fluctuating two-component field. The space distribution of the difference $A(\mathbf{r})=1-|\varphi(\mathbf{r})|$ is shown by a surface of a constant level corresponding to the $A(\mathbf{r})=0.05\left|\boldsymbol{\varphi}_{0}\right|$ (where $\left|\boldsymbol{\varphi}_{0}\right|$ denotes a modulus of the two-component field in an equilibrium $\left|\varphi_{0}\right|=1$ ). 


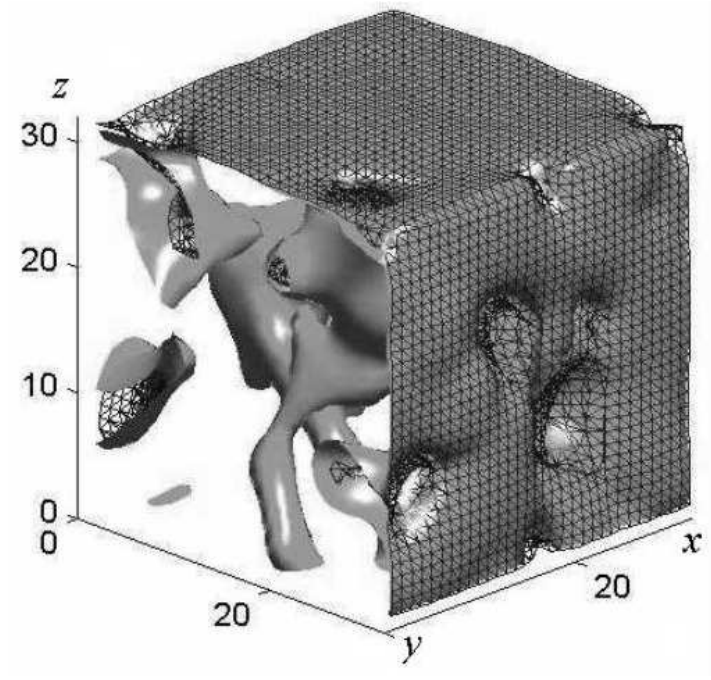

Figure 6. An illustration to a mutual relation between the toroidal peculiarities of the order parameter field and "usual" vortex-antivortex pairs (a symbolic cross-section of them by the planes $z=$ const, $y=$ const).

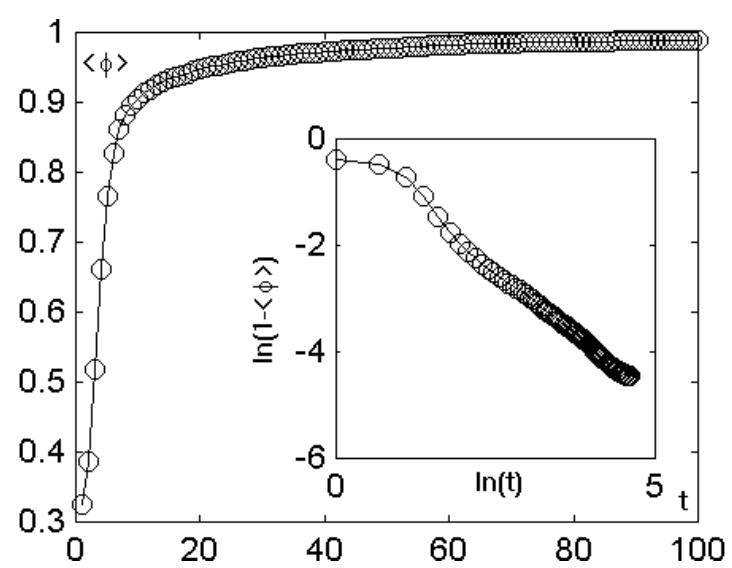

Figure 7. A time dependence of the mean order parameter $\langle\varphi\rangle$ for the twocomponent system. In the insert, the same dependence is presented in a form of double logarithmic plot.

that is convenient for the numerical calculation. An evolution of the dissipation rate $W$ obtained in such a way is shown in figure 4 .

To exclude an impact of the quick random processes, this calculation has been performed for the case $\zeta\left(\mathbf{r}_{j}, t\right)=0$. We start from a random distribution of the order parameter field to create the super-critical nuclei. The system quickly suppresses short-wave fluctuations. It is reflected by a fast decrease of the curve $W(t)$ at small $t$. There is a relation between the behaviour of the maxima $R$ and the dissipation $W$. When the nuclei grow quickly and form the structure, the dissipation is relatively high. Later, when the structure has already been formed and the only expansion of the filamentary domains takes place, the dissipation tends to a minimum. It agrees with the results by general kinetic theory [32-34] stating that the intermediate structure is an attractor for the fluctuating field $\varphi(\mathbf{r}, t)$.

The increase of the component number to the complex field does not change this result. So, let us concentrate on the qualitatively new results only. The components fluctuate independently and produce their own domain structures. But, these structures interact and, as result, they are correlated. The interaction of the component is presented in the fourth order terms of the free energy density (5). So, it is "switched on" at relatively late stages of the ordering, when both of the components are close to the equilibrium values inside the domains. But, there is no difference in the energy for the domains with a positive and a negative order parameter. So, this contains both types of the domains as well as a lot of regions between them, where the modulus $|\boldsymbol{\varphi}(\mathbf{r})|^{2}$ falls down to zero. This state is well known for the 2D systems. It is the so-called Berezinsky-Kosterlitz phase [42-45]. Normally it is accompanied 
by a formation of the vortex pairs.

A part of such an intermediate phase in 3D-space is shown in figure 5 . The difference $A(\mathbf{r})=1-|\boldsymbol{\varphi}(\mathbf{r})|$ is shown in this figure by means of. the surface of constant $A(\mathbf{r})$ value: $A(\mathbf{r})=0.05\left|\boldsymbol{\varphi}_{0}\right|$. There is some relation between planar vortex pairs and 3D-toroids shown in figure 5 . It is shown in a small fragment of the system presented in figure 6 .

This toroidal state is a long-time living structure from the kinetic point of view. Quantitatively it can be characterized by a deviation of a mean instant order parameter $\langle|\boldsymbol{\varphi}|\rangle=\int \mathrm{d}^{\mathrm{d}} \mathbf{r}|\boldsymbol{\varphi}(\mathbf{r})| 1 / V$ from the equilibrium value $\left|\boldsymbol{\varphi}_{0}\right|=1$.

A corresponding time dependence is shown in figure 7 . It is seen from a doublelogarithmic plot in the insert to figure 7 that (starting from some moment) this evolution is logarithmically slow. It corresponds to a slow relaxation of the structure containing the "frozen" toroidal vortices. The system reduces a gradient energy by a straightening of the domain boundaries. Their mutual intersection goes down and the torus diminish. When the intersection disappears the torus "annihilates". In 2D cross-sections of the torus one can observe an "annihilation" of the vortex-antivortex pair. Just before the annihilation, the small torus becomes very close to an ideal circular one. The couple of small circular torus (marked by the rectangulars) can be observed in figure 5 .

\section{References}

1. Landau L.D., Lifshits E.M. Statistical Physics. Moscow, Nauka, 1988.

2. Landau L.D., Pitaevsky L.D. Physical Kinetics. Moscow, Nauka, 1979.

3. Loskutov A.Yu., Mikhailov A.S. Introduction to the Sinergetics. Moscow, Nauka, 1990.

4. Velasco E., Toxyaerd S. // Phys. Rev. Lett., 1993, vol. 71, p. 388.

5. Alexander F.J., Chen S., Grunau D.W. // Phys. Rev. B, 1993, vol. 48, p. 634.

6. Leptoukh G., Strickland B., Roland C. // Phys. Rev. Lett., 1995, vol. 74, p. 3636.

7. Bastea S., Lebowitz J.L. // Phys. Rev. Lett., 1995, vol. 75, p. 3776.

8. Wei D., Patey G.N. // Phys. Rev. Lett., 1992, vol. 68, p. 2043.

9. Weis J.J., Levesque. // Phys. Rev. Lett., 1993, vol. 71, p. 2729.

10. Ayton J., Gingras M.I.P., Patey G.N. // Phys. Rev. Lett., 1995, vol. 75, p. 2360.

11. Svishchev I.M., Kusalik P.G. // Phys. Rev. Lett., 1994, vol. 73, p. 975.

12. Svishchev I.M., Kusalik P.G. // Phys. Rev. Lett., 1995, vol. 75, p. 3289.

13. Kukharkin N., Orszag S. A., Yakhot V. // Phys. Rev. Lett., 1995, vol. 75, p. 2486.

14. Smith L.M., Yakhot V. // Phys. Rev. Lett., 1993, vol. 71, p. 352.

15. Fine K.S. et al. // Phys. Rev. Lett., 1995, vol. 75, p. 3277.

16. Aharony A. - In: Phase Transitions and Critical Phenomena. New York, Academ Press, 1976.

17. Izumov Yu.A., Skryabin Yu.N. Statistical Mechanics of the Magnetically Ordered Systems. Moscow, Nauka, 1987 (in Russian).

18. Patashinsky A.Z., Pokrovsky A.Z. Fluctuation Theory of Phase Transitions. Moscow, Nauka, 1982 (in Russian).

19. Ivanchenko Yu.M., Lisyansky A.A., Filippov A.E. Fluctuation Effects in the Systems with Competitive Interactions. Kiev, Naukova Dumka, 1989 (in Ukrainian). 
20. Khachaturyan A.G. Theory of the Phase Transitions and the Structure of Solid Solvents. Moscow, Nauka, 1974 (in Russian).

21. Landau L.D., Khalatnikov L.D. Collected Works of Landau L.D. Moscow, Nauka, 1969, vol. 2, p. 218.

22. Allen S.M., Cahn J.W. // Acta Metall., 1979, vol. 27, p. 1085.

23. Cahn J.W. // Acta Metal., 1960, vol. 8, p. 554.

24. Filippov A.E. // JETP, 1997, vol. 84, p. 971.

25. Mazor A., Bishop A.R. // Physica D., 1989, vol. 39, p. 22.

26. Falk F. // Z. Phys. B., 1984, vol. 54, p. 159.

27. Falk F. // J. Phys. C: Solid State Phys., 1987, vol. 20, p. 2501.

28. Rao M., Chakrabarti A. // Phys. Rev. Lett., 1993, vol. 71, p. 3501.

29. Kuklin R.N., Kuzmin V.N. // Fiz. Tverd. Tela., 1991, vol. 33, p. 2401.

30. Rogers T.M., Elder K.R., Desai R.C. // Phys. Rev. B., 1988, vol. 37, p. 9638.

31. Elder K.R., Desai R.C. // Phys. Rev. B., 1989, vol. 40, p. 243.

32. Kuzovlev Yu.E., Soboleva T.K., Filippov A.E. // JETP Lett., 1993, vol. 58, p. 357.

33. Kuzovlev Yu.E., Soboleva T.K., Filippov A.E. // JETP Lett., 1993, vol. 76, p. 858.

34. Filippov A.E., Kuzovlev Yu.E., Soboleva T.K. // Phys. Lett. A, 1992, vol. 165, p. 159.

35. Zeltser A.S., Filippov A.E. // JETP, 1994, vol. 79, p. 605.

36. Zeltser A.S., Soboleva K., Filippov A.E. // JETP, 1995, vol. 81, p. 193.

37. Filippov A.E. // J. Stat. Phys., 1994, vol. 75, p. 241.

38. Filippov A.E. // Phys. Lett. A, 1998, vol. 243, p. 229.

39. Filippov A.E., Radievsky A.V., Zeltser A.S. // Phys. Lett. A, 1994, vol. 192, p. 131.

40. Geim A.K., Grigorieva I.V., Dubonos S.V., Lok J.G.S, Maan J.C., Filippov A.E., Peeters F.M. // Nature, 1997, vol. 390, p. 259.

41. Filippov A.E., Radievsky A.V., Zeltser A.S. // Phys. Rev. B, 1996, vol. 54, p. 3504.

42. Berezinsky V.L. // ZhETF, 1970, vol. 59, p. 907; ZhETF, 1971, vol. 60, p. 1144.

43. Kosterlitz J.M., Tauless D.J. // J. Phys. C: Solid State Phys., 1973, vol. 6, p. 1181.

44. Kosterlitz D.J. // J. Phys. C: Solid State Phys., 1974, vol. 7, p. 1046.

45. Minnhagen P. // Rev. Mod. Phys., 1987, vol. 59, p. 1001. 


\section{Чисельне моделювання 3-вимірних структур флуктуюючих полів}

\section{О.Є.Філіппов}

Донецький фізико-технічний інститут, 83114, Донецьк

Отримано 8 лютого 2001 р.

Чисельне моделювання структури флуктуюючого поля виникаючого на рівні кінетичного опису фазового переходу здійснено для систем з скалярним і дво-компонентним параметрами порядку. Виявлено, що процес впорядкування супроводжується велико-масштабною волокнистою структурою флуктуюючого поля. В системі утворюються густі локальні групи і ланцюжки ядер. Виявлено складну топологію впорядкування в системі з двокомпонентним параметром порядку. Така система утворює складні тороїдальні завихрення. Кінетичний сценарій в 3-вимірному просторі порівнюється з попередньо одержаними дво- і одновимірними проекціями аналогічних структур.

Ключові слова: фазові переходи, флуктуації, нуклеація, кінетика

PACS: $64.60 . A k, 64.60 . Q b$ 